\title{
Gonococcal infections: The trends of antimicrobial susceptibility of Neisseria gonorrhoeae in Western Nepal
}

\author{
Bhatta DR, ${ }^{1^{*}}$ Gokhale S, ${ }^{1}$ Ansari MT, ${ }^{1}$ Tiwari HK, ${ }^{1}$ Gaur A, ${ }^{1}$ Mathuria $J P,{ }^{1}$ Ghosh $A N^{1}$ \\ ${ }^{l}$ Department of Microbiology, Manipal College of Medical Sciences, Pokhara, Nepal.
}

\author{
*Corresponding Author: \\ Dharm Raj Bhatta, \\ Department of Microbiology, \\ Manipal College of Medical Sciences, \\ Phulbari, Pokhara, Nepal. \\ Email: ddharma2039@gmail.com
}

\section{Citation}

Bhatta DR, Gokhale S, Ansari MT, Tiwari HK, Gaur A, Mathuria JP, Ghosh AN. Gonococcal infections: The trends of antimicrobial susceptibility of Neisseria gonorrhoeae in Western Nepal. Nepal Journal of Medical sciences 2012;1(2):74-8.

\begin{abstract}
Background: Gonorrhea is caused by Neisseria gonorrhoeae, is an important public health problem and is the second most common reportable sexually transmitted bacterial infection. Present study was conducted to determine the antimicrobial resistance pattern of Neisseria gonorrhoeae isolates from various clinical specimens.
\end{abstract}

Methods: This is a hospital based retrospective study conducted at Manipal Teaching Hospital, Pokhara, Nepal. Various clinical specimens (urethral, cervical and conjunctival discharges) were collected from the suspected cases of gonococcal infections between January 2004 to December 2010. Specimens were subjected to Gram stain and culture on chocolate agar. Antibiotic susceptibility testing was performed on chocolate agar by Kirby Bauer's disc diffusion method.

Results: A total of 119 patients were tested for gonococcal infections. Forty-eight patients were diagnosed as having gonococcal infections, of which 40 cases were culture positive. Penicillin resistance was seen in 27 (67\%) cases while all isolates were sensitive to ceftriaxone.

Conclusion: Neisseria gonorrhoeae isolates are becoming increasingly resistant to antibiotics like penicillin, ciprofloxacin and tetracycline. Therefore, continuous surveillance of antibiotic resistance pattern is required in order to start empirical antibiotic therapy in high risk population like commercial sex workers.

Keywords: Antimicrobial susceptibility; gonorrhoeae; N. gonorrhoeae; urethritis.

\section{Background:}

Sexually transmitted infections are infections that are spread primarily through person-to-person sexual contact. Gonorrhea is a sexually transmitted disease caused by the bacterium Neisseria gonorrhoeae and humans are the only natural host. It is a curable sexually transmitted disease (STD) but if left untreated can lead to complications such as infertility, arthritis, meningitis and septicemia. It mainly inhabits the genital area and survives poorly outside the human body. Neisseria gonorrhoeae causes infections principally of the urethra in men and the endocervix in women, although it may also infect extra genital mucosal sites, including the oropharynx and anorectum. Ocular infections can also occur, and in neonates can cause blindness due to ophthalmia neonatorum. Genital infection in men 
usually presents with urethral discharge, but asymptomatic infections are common among women.

Gonorrhoeae remains a significant disease globally. The prevalence of gonorrhea is not uniform and varies greatly among developed and developing countries. Center for Disease Control and Prevention (CDC) estimates that more than 700,000 persons in the US get new gonococcal infection each year. Less than half of these infections are reported to CDC. In 2007, reported cases of gonorrhea to the CDC were 356,524 , while in 2009, approximately 301,000 cases of gonorrhoea were reported. ${ }^{1,2}$ According to Family Health Division, Ministry of Health, Nepal, gonorrhea is one of the most prevalent STD in Nepal. ${ }^{3}$ This problem is further complicated by the emergence of drug resistant strains of $N$. gonorrhoeae to commonly used antimicrobial agents, making the treatment expensive and prolonged. Various factors which influence the incidence of gonorrhea include pattern of sexual behavior, population demographics, and economic and social conditions.

The emergence of strains of $N$. gonorrhoeae, resistant to various antimicrobial agents due to widespread dissemination of resistant clones and the emergence of strains with novel resistance mechanisms, is a major obstacle in the disease management. ${ }^{4}$ Appropriate and effective treatment requires updated information about susceptibility patterns. Because of the emergence of drug resistant strains like penicillinase producing $N$. gonorrhoeae (PPNG), plasmid-mediated tetracycline resistance (TRNG), and chromosomally mediated resistance to penicillin and/or tetracycline (CMRNGPT), the Centers for Disease Control and Prevention, Atlanta, has advocated the use of expandedgeneration cephalosporin or fluoroquinolones as the first line of therapy for uncomplicated gonorrhea. ${ }^{5}$

\section{Methods:}

This retrospective cross sectional study was conducted in Department of Microbiology, Manipal Teaching Hospital, Pokhara, Nepal. A total of 119 patients attending Venerology and Gynecology OPD between January 2004 to December 2010 with complaints of urethritis, cervicitis and neonatal conjunctivitis were included in this study. Brief clinical history of patients regarding unprotected sexual contact with the professional sex workers and multiple sex partners was recorded. All the patients were additionally screened for Human Immunodeficiency Virus (HIV) by Rapid test kit (Tridot J. Mitra \& Co. Pvt. Ltd) and Syphilis by RPR test (Trepostat, Ranbaxy).
Urethral discharge, cervical and conjunctival swabs were collected from the patients as per clinical presentations. Smears were Gram stained and examined for presence of pus cells and intracellular Gram negative diplococcic (ICDC). Urethritis and cervicitis were diagnosed when $>$ $4 \mathrm{PMNL} /$ field and $>30 \mathrm{PMNL} /$ field were seen along with ICDC respectively. ${ }^{6}$ Specimen was directly inoculated on chocolate agar and incubated in candle jar at $37^{\circ} \mathrm{C}$ upto 48 hrs. Isolates were identified based on colony morphology, Gram staining, oxidase test, and rapid carbohydrate utilization test. Antimicrobial susceptibility test was done on chocolate agar by Kirby Bauer method. The antibiotic discs used were penicillin (10 I.U.), ciprofloxacin (5 mcg), tetracycline $(30 \mathrm{mcg})$ and ceftriaxone $(30 \mathrm{mcg})$.

\section{Results:}

A total of $40 \%(48 / 119)$ patients were diagnosed to have gonococcal infection. A total of $40 N$. gonorrhoeae strains were isolated. Out of total 48 cases, 36 were diagnosed by both microscopy and culture while 4 were culture positive but Gram stain negative. Out of total 48 cases, 8 were diagnosed by microscopic examination only. The year wise distribution of number of patients tested for gonorrhoea with the results of gram stain and culture is summarised in Table 1.

Table 1: Year wise distribution of the patients, and results of gram stain and culture:

\begin{tabular}{cccc}
\hline Year & $\begin{array}{c}\text { Number of } \\
\text { patients }\end{array}$ & $\begin{array}{c}\text { Gram stain (ICDC) } \\
\text { Positive }\end{array}$ & $\begin{array}{c}\text { Culture } \\
\text { positive }\end{array}$ \\
\hline 2004 & 25 & 13 & 10 \\
2005 & 23 & 06 & 07 \\
2006 & 21 & 06 & 08 \\
2007 & 20 & 08 & 06 \\
2008 & 17 & 06 & 04 \\
2009 & 08 & 03 & 03 \\
2010 & 05 & 02 & 40 \\
\hline Total & 119 & 44 & 02 \\
\hline
\end{tabular}

Majority of patients were male, between (15-25) years of age. Age and gender wise distribution of the patient is summarized in Table 2. 
Table 2: Age and gender wise distribution of the patients:

\begin{tabular}{ccc}
\hline Age (Years) & Male & Female \\
\hline Neonate & 04 & 01 \\
$15-25$ & 44 & 01 \\
$26-35$ & 34 & 05 \\
$36-45$ & 19 & 03 \\
$46-55$ & 06 & 00 \\
$>55$ & 02 & 00 \\
Total & 109 & 10 \\
\hline
\end{tabular}

Total cases of gonococcal infection: 48

Gonococcal Ophthalmia neonatorum: $04(8 \%)$

Gonococcal urethritis (Male): 42 (88\%)

Gonococcal cervicitis (Female): 02 (4\%)

About 17\% (20/105) of the patients were partially treated before reporting to our hospital. Purulent urethral discharge was the commonest symptom in male. Among the male gonorrhea cases, co-infection with HIV was reported in $6 \%$ followed by syphilis $4 \%$. Seventy five percent (36/48) gave history of unprotected sexual contact with commercial female sex workers. The source of infection in female could not be ascertained. In neonates the source of infection was maternal genital canal.

Out of total 40 isolates, only $13(32 \%)$ were found to be sensitive to penicillin, 23 (57\%) isolates were sensitive to Ciprofloxacin, while 22 (55\%) were sensitive to Tetracycline. All the isolates were sensitive to Ceftriaxone. Table 3 summarizes the antibiogram of $N$. gonorrhoeae isolates.

Table 3: Antibiotic susceptibility pattern of $N$. gonorrhoeae (2004-2010):

\begin{tabular}{lcc}
\hline Antibiotic & Sensitive (\%) & Resistant (\%) \\
\hline Ceftriaxone & $40(100 \%)$ & 00 \\
Ciprofloxacin & $23(57.5 \%)$ & $17(42.5 \%)$ \\
Penicillin & $13(32.5 \%)$ & $27(67.5 \%)$ \\
Tetracycline & $22(55 \%)$ & $18(45 \%)$ \\
\hline
\end{tabular}

Most of the penicillin resistant isolates were from the patients of age group 15 to 25 years $(85 \%)$, followed by 26 to 35 years $(67 \%)$, neonates $(50 \%)$ and 36 to 45 years $(33 \%)$ as shown in Table 4.

Table 4: Age-wise distribution of penicillin resistant isolates

\begin{tabular}{cccc}
\hline $\begin{array}{c}\text { Age group } \\
\text { (Years) }\end{array}$ & $\begin{array}{c}\text { Total } \\
\text { isolates }\end{array}$ & $\begin{array}{c}\text { Penicillin } \\
\text { resistant } \\
\text { isolates }\end{array}$ & Percentage \\
\hline Neonate & 04 & 02 & 50 \\
$15-25$ & 20 & 17 & 85 \\
$26-35$ & 12 & 08 & 66 \\
$36-45$ & 03 & 01 & 33 \\
$46-55$ & 01 & 00 & 00 \\
$>55$ & 00 & 00 & 00 \\
\hline
\end{tabular}

Temporal pattern of penicillin resistance of $N$. gonorrhoeae is summarized in the Figure 1.

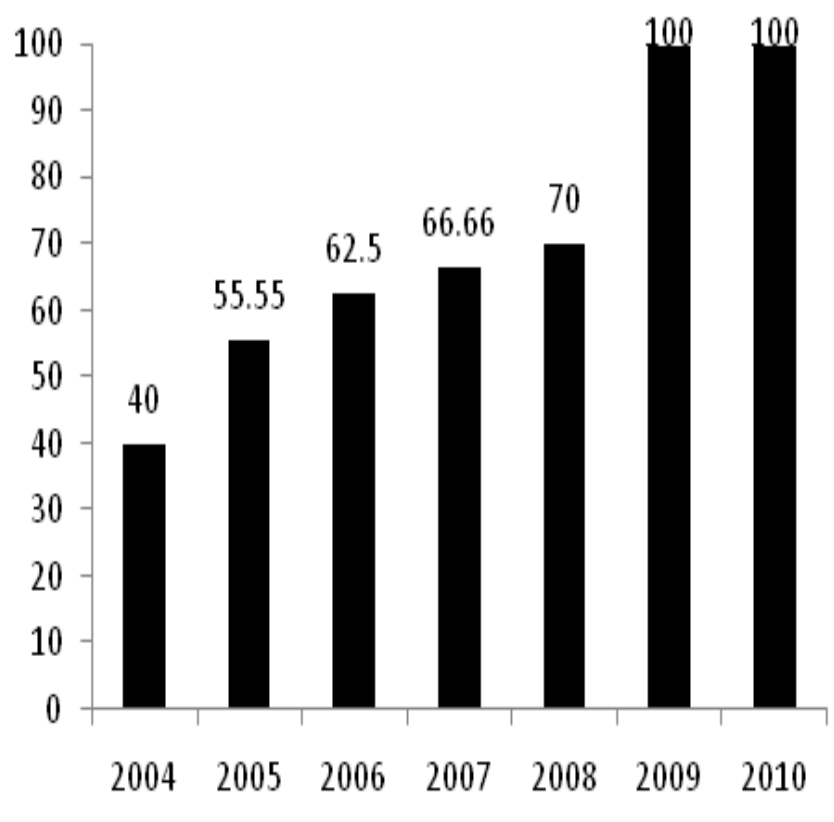

Figure: 1 Penicillin resistant $N$. gonorrhoeae isolates (2004-2010)

\section{Discussion:}

Out of 119 suspected cases, gonococcal infections were confirmed in only 48 cases by laboratory findings. Majority of the patients were between the age group 15-25 year and unmarried, hence more prone to visit commercial sex 
workers. Similar findings have been reported by other workers. ${ }^{7}$ In our study, penicillin resistance was found to be $67.5 \%$ which was higher than the study from Tribhuvan University Teaching Hospital (TUTH) in 2001. ${ }^{8}$ Similar study from Birjung, Nepal, reported penicillin resistance in $60 \%$ of the isolates which was less than our study. ${ }^{9}$ Our study highlights the increasing rate of penicillin resistant gonococci in the Western Region of Nepal.

It is interesting to see the remarkable increase in the penicillin resistance of $N$. gonorrhoeae isolates from the year 2004 to 2010. Penicillin resistance in the year 2004 was only $40 \%$; in the year 2005 , it increased up to $55 \%$. Then there was gradual rise in the resistance; $62 \%$ in $2006,67 \%$ in $2007,70 \%$ in 2008 , peaking to $100 \%$ in 2009 . The same trend continued in 2010 also.

Though the number of gonorrhea cases has shown downward trend, resistance to penicillin has shown upward trend from 2004-2010. Increased awareness among general population as well as sex workers regarding Sexually Transmitted Diseases and increased practice of protected sexual intercourse could be the possible factors associated with decreasing number of cases of gonorrhea. This high rate of penicillin resistance in the Western part of Nepal may be attributed to the low socio-economic status, lack of appropriate medical facilities and partial treatment at outside clinics. Indiscriminate use of antibiotics and delay in seeking medical treatment could be other reason for high rate of penicillin resistance.

Manipal Teaching Hospital (MTH) is a tertiary care centre of Western Nepal. Majority of patients usually take the initial treatment in primary health care centers/General practioners before reporting to our center. Patients usually come to MTH when the disease becomes chronic and this could be another possible reason for higher percentage of drug resistance in our isolates. Present study also highlights the gradual decrease in the incidence of gonorrhoeae in the Western Region. In the year 2004, number of patients visiting Skin and STD OPD with complaint of urethritis was 24 , of which 13 were diagnosed to have gonococcal urethritis. From the year 2005 onwards, continuous decline in the number of patients and positivity cases was observed.

The increasingly widespread use of nonculture methods for diagnosis is a major challenge for monitoring resistance pattern. ${ }^{10}$ Furthermore, routine culture and antimicrobial susceptibility testing is not performed in rural areas because of high cost, poor laboratory facilities and inadequate quality controls. The empirical therapy of gonococcal urethritis should be guided by continuous surveillance of the antimicrobial susceptibility. Due to absence of adequate laboratory data and an established monitoring system, selection of appropriate antibiotics for empirical treatment of gonorrhea is difficult. Present study highlighted the highlevel resistance to penicillin, tetracycline, and ciprofloxacin, indicating that these antimicrobials are not suitable for empirical treatment of gonorrhea.

In our study, all isolates of $N$. gonorrhoeae were found to be sensitive to Ceftriaxone. Therefore, Ceftriaxone should be considered as the drug of choice for the treatment of gonorrhoeae. Center for Disease Control and Prevention (CDC) has recommended single dose therapy with a broad spectrum cephalosporin (Cefipime or Ceftriaxone) or a fluoroquinolone (Ciprofloxacin or Ofloxacin) for uncomplicated gonorrhea. ${ }^{11}$

Co-infection rate with $H I V$ in our study was $6 \%$ which is slightly higher than the study from India, ${ }^{7}$ possibly because of low sample size for analysis in our study.

\section{Conclusion:}

Penicillin resistance among $N$ gonorrhoeae isolates is very high, Periodic monitoring of antimicrobial susceptibility of $N$ gonorrhoeae is essential for early detection of emergence of drug resistance. Ceftriaxone should be considered as drug of choice for empirical treatment of gonococcal urethritis.

Acknowledgement: We would like to thank all the staff of Microbiology Department, Manipal Teaching Hospital, Pokhara, Nepal for their co-operation.

\section{Conflict of interest: none}

\section{References:}

1. Centers for Disease Control and Prevention. Sexually Transmitted Disease Surveillance, 2009. Atlanta, GA: U.S. Department of Health and Human Services, November 2010.

2. Centers for Disease Control and Prevention. Sexually Transmitted Diseases Treatment Guidelines, 2010. MMWR 2010;59 (no. RR-12).

3. Ministry of Health, Government of Nepal. National Medical Standard for Reproductive Health Services. Family Health Division, August 2003.

4. Ison CA, Pepin NS, Roope NS, et al. The dominance of a multiresistant strain of Neisseria gonorrhoeae among prostitutes and STD patients in the Gambia. Genitourinary Med 1992;68:356-60. 
5. Workowski KA. The 1998 CDC sexually transmitted diseases treatment guidelines. Curr Infect Dis Rep 2000;2:44-50.

6. Mandell GL, Bennett JE, Dolin R. Principles and practice of Infectious Diseases. $4^{\text {th }}$ ed. USA: Churchill Livingstone. 1995:1074-85.

7. Manju B, Jhinuk BM, Sumathi M, et al. Gonorrhoea $\&$ its co-infection with other ulcerative, non-ulcerative sexually transmitted \& HIV infection in a Regional STD Centre. Indian J Med Res 133,2011;346-9.

8. Ray K, Bala M, Kumari S, et al. Antimicrobial resistance of Neisseria gonorrhoeae in selected World Health Organization Southeast Asia Region Countries. An overview. Sex Transm Dis 2005;32:178-84.

9. Bhargava D, Shakya B, Mondal KC, et al. Emergence of penicillin resistant Neisseria gonorrhoeae. J Inst Med 2010;32:15-18.

10. Knapp JS. Neisseria gonorrhoeae resistant to ciprofloxacin and ofloxacin. Sex Transm Dis 1998;25:425-6.

11. Sexually transmitted diseases treatment guidelines. MMWR Morb Mortal Wkly Rep.1993;42:57-65. 